\section{A rapid, low-cost methodology for longitudinal evaluation of insecticide treated bednet distributions: a pilot survey coupling bednet usage with malaria prevalence at the household level}

\author{
Karen B. Jacobson, ${ }^{1}$ Dennis Danforth, ${ }^{1}$ \\ James Lin, ${ }^{1}$ Steven Merjavy, ${ }^{1}$ \\ Brendan Milliner, ${ }^{1}$ Kohtaro Ooka, ${ }^{1}$ \\ Nils Hennig² \\ 'Icahn School of Medicine at Mount Sinai, \\ New York; \\ ${ }^{2}$ Global Health Center, Mount Sinai \\ School of Medicine, New York, NY, USA
}

\section{Abstract}

Tanzania's National Malaria Control Program distributed 23.3 million insecticidetreated bed nets (ITNs) between 2009 and 2011. Annual randomized household surveys were conducted from 2009 to 2011 to assess the incremental effects of the distribution campaign on malaria prevalence and bednet usage in Kijumbura village in Kagera, Tanzania. Data was collected about household ITN ownership and individual use, and each household member was given a rapid malaria diagnostic test (RDT). In total, 1247 individuals from 285 households participated. From 2009 to 2011, household ITN ownership increased from 50.6 to $95.3 \%$ and individual usage increased from 9.7 to $55.3 \%$ in 2011 . Malaria point prevalence decreased from $15.8 \%$ in 2009 to $6.5 \%$ in 2010 , and increased from $6.5 \%$ in 2010 to $10.7 \%$ in 2011 . The survey cost in 2011 was 23.50 USD per household; major expenses were transportation, personnel payment, and the purchase of the RDTs. Evaluations of bednet distribution programs generally rely on distribution data and selfreported net ownership, but it is important to also assess the goal endpoint of reduction in malaria prevalence. We show that this can be achieved quickly and cost-effectively through randomized surveys measuring bednet usage coupled with malaria prevalence at the household level.

\section{Introduction}

Malaria persists as the most common public health problem and a major cause of morbidity and mortality in Tanzania, with 3.8 million probable or confirmed cases in 2009. ${ }^{1}$ Large international donors such as the President's Malaria Initiative, the Global Fund to Fight AIDS, Tuberculosis, and Malaria, and the Bill and Melinda Gates Foundation have been partnering with national governments in malaria endemic nations to deploy insecticide-treated nets (ITNs), indoor residual spraying (IRS), and artemisinin combination therapy (ACT) distribution programs.

Tanzania's National Malaria Control Program (NMCP), with the help of the United States Agency for International Development (USAID), has implemented programs to scaleup ITN usage. The Tanzania National Voucher Scheme (TNVS) is a public-private partnership launched in 2004 that offers highly subsidized ITNs to pregnant women and caregivers of infants through vouchers given out at antenatal clinics and redeemable at local places that sell ITNs. However, by 2008 only a quarter of pregnant women and children younger than 5 were using ITNs and many of the nets that were distributed needed to be re-treated with insecticide every 6 months. The NMCP decided to begin providing long-lasting insecticidal nets (LLINs) in the voucher scheme, reduced the voucher price, and initiated a campaign to distribute free LLINs to all children under age five. From August 2009 to May 2010, the NMCP distributed 8.7 million LLINs to households with children under five years old or pregnant women. ${ }^{2}$ This plan was expanded further with the announcement of a policy goal of universal LLIN coverage, defined as one LLIN per sleeping space. An estimated 14.6 million LLINs have been distributed under this policy. ${ }^{2}$ In Tanzania, the Under 5 Coverage Campaign and the Universal Coverage Campaign together will cost an estimated 150 million USD for the distribution of almost 27 million ITNs. ${ }^{2}$

Although the benefit of ITNs and IRS as preventative measures against malaria is wellestablished and sub-Saharan African countries have seen a substantial decline in malaria over the past decade, it is difficult to properly evaluate the efficacy of individual malaria vector control programs. Most data concerning ITN/LLIN distributions are obtained from suppliers and focus on the number of nets distributed, for example via voucher tracking, ${ }^{3,4}$ with little information about household ownership and usage in the distribution areas before and after distribution, because ownership and usage at a household level are difficult to assess frequently enough for meaningful analysis. When ownership and usage data are obtained periodically through household surveys, it is usually only every $3-5$ years, and mathematical models integrating distribution and survey data are used to estimate ownership and usage statistics. ${ }^{5}$ Furthermore, household surveys assessing bednet usage often do not account for malaria parasitemia in the households surveyed. ${ }^{6,7}$ Cost and time are
Correspondence: Karen B. Jacobson, Icahn School of Medicine at Mount Sinai, 1428 Madison Ave., 10029 New York, NY, USA.

E-mail: karen.jacobson@mssm.edu

Key words: Malaria, Tanzania, insecticide treated nets.

Contributions: JL and SM designed the study and collected data; DD and BM contributed to data collection and analysis; KO and KBJ collected and analyzed data and finalized the manuscript; $\mathrm{NH}$ served as faculty advisor for the project.

Conflict of interests: the authors declare no potential conflict of interests.

Acknowledgements: this project was made possible through the generous support of the Mount Sinai Global Health Center and the Arnold P. Gold Foundation Student Summer Fellowship. The authors would like to acknowledge Johansen Ernest, our operations coordinator without whom this project would not have been possible. Additional thanks to Lynus, Ndinda, Marigoreth, and Prisca. Finally, a sincere thanks to the residents and hamlet leaders of Kijumbura, Tanzania.

Received for publication: 11 July 2013.

Accepted for publication: 4 February 2014.

This work is licensed under a Creative Commons Attribution NonCommercial 3.0 License (CC BYNC 3.0).

(C) Copyright K. B. Jacobson et al., 2014

Licensee PAGEPress, Italy

Malaria Reports 2014; 3:1823

doi:10.4081/malaria.2014.1823

often cited as the reason for lack of more rigorous study designs in evaluating the efficacy of malaria control programs. ${ }^{8}$

Other methods that have been employed to assess bednet usage and bednet distribution programs include vector measurement and measurement of mortality rates. ${ }^{9}$ However, vector measurement is time-consuming and costly, and mortality rates are not indicative of a direct effect of vector control measures on malaria incidence. While these strategies may yield valid measures for assessing bednets as a preventive measure or evaluating the efficacy of a particular distribution program's design, they are too time-consuming and costly to perform on a wide scale and repeatedly on a regular basis.

Following the NMCP's distribution of LLINs to households with children under five, an English academic institute and an evaluation contractor conducted a household survey of ITN usage in selected areas, ${ }^{10}$ compared to baseline data from the last national survey completed in 2008. ${ }^{11}$ Although the post-inter- 
vention monitoring program measured household ownership and usage of bednets after the intervention, no data were collected about malaria prevalence in the households and communities where the survey took place. Total expenditure on monitoring during the Under 5 Coverage Campaign was 106,000 USD which paid for survey of 4500 households (24.44 USD per household). ${ }^{10}$

In 2007, researchers from Mount Sinai School of Medicine in New York developed a plan for a rapid yet detailed assessment of the progress of the NMCP's malaria control program in one village while limiting on-site personnel to medical students, local nurses, and local lay people. Researchers assessed baseline malaria prevalence and bednet usage at the household level and repeated the study annually to investigate changes in these parameters following two phases of malaria control measures implemented by the NMCP.

\section{Materials and Methods}

Researchers completed a baseline pre-distribution assessment in July 2009. A second assessment was completed in 2010 following the distribution of LLINs to households with children under five years old. The third and final assessment of malaria prevalence and bednet usage was completed in July 2011, after the NMCP completed universal distribution of LLINs. The survey protocol, data collection tool and informed consent documents were reviewed and approved by the Institutional Review Board at the Mount Sinai School of Medicine and Tanzania's National Institute for Medical Research.

\section{Village setting and selection of households}

Kijumbura village is located in the Karagwe district of northwestern Tanzania near the border with Rwanda. Far from academic institutions and cities, Kijumbura is a rural village of approximately 6700 living in 1400 households spread over 9 hamlets. Researchers collaborat- ed with the village chairman and hamlet leaders, who provided a list of all households by hamlet numbered in roughly geographic order moving from north to south. Households were selected using systematic random sampling; a random number generator was used to select the first household, and thereafter every seventeenth household was selected from the lists as calculated for a minimum goal of 85 households per survey year. Local residents acted as hamlet guides and assisted with navigation.

\section{Data collection}

Each of the three phases of the survey was carried out over 7-9 days, during which approximately 400 individuals each year were tested for malaria using rapid diagnostic tests (RDTs). Written informed consent was obtained both from community leaders and from individual participants or guardians of participants under 18 years at the time of data collection. Participants under 18 years gave verbal assent in addition to the written informed consent of their guardian. Consent documents were translated into Swahili and back-translated to ensure accuracy.

Two teams - each consisting of a local nurse, translator, hamlet guide and medical student researcher - conducted the survey. Each team was assigned one area of a hamlet to cover per day. Translators obtained written consent from each head of household. If the household did not consent - a rare occurrence - or if no one was present, teams proceeded to the next household on the hamlet list.

After consent, the nurse administered the rapid diagnostic test (RDT) to all present members of the household. There were no exclusion criteria; all present and consenting members of selected households were allowed to participate. The First Response Malaria Antigen pLDH/HRP2 Combo (Premier Medical Corp., Watchung, NJ, USA) was chosen for this study because it had been field tested successfully and also performed well in the first and second rounds of the WHO Malaria RDT Product Testing program. ${ }^{12}$ Meanwhile the translator collected information on basic demographics, history of bednet and malaria education, household net ownership, fever history, and bednet usage for each member of the household. Survey questions were adapted from the WHO Malaria Indicator Survey Household Questionnaire. ${ }^{13}$ The survey was translated into Swahili and back-translated to ensure accuracy. The researchers directly observed whether a net hung over each bed. Axillary temperatures were taken with a digital thermometer. Children under 18 who tested positive for malaria were treated with weight appropriate dose of Coartem (Novartis Pharma AG, Basel, Switzerland). Adults who tested positive were treated if they had a body temperature above $37^{\circ} \mathrm{C}$ or reported a history of subjective fever in the previous 3 days. Primary outcome measures were positive malaria rapid diagnostic test, number of nights spent under net in past week, and number of bednets owned by household. Secondary outcomes were gender, age, pregnancy status, temperature, history of fever in past 3 days, and household head having received bednet education.

\section{Data analysis}

The data was analyzed using Microsoft Excel 2010 for Windows and STATA/SE 11.0. Summary statistics for each year were obtained, and chi-squared tests were performed to compare malaria prevalence, household net ownership, and bednet usage across the three years.

\section{Results}

The project was successful in measuring malaria status and bednet usage rates for 1247 individuals from 285 households in a remote area of northwest Tanzania. Thirty-five participants (2.8\%) were pregnant women and 313 (25.1\%) were under five years of age. Researchers encountered 443 nets of which 217 had been used the previous night. One hundred thirty one participants (10.5\%) were treated for malaria. Individual net usage increased each year from $20.4 \%$ in 2009 to $38.4 \%$ in 2010 and then to $65.8 \%$ in 2011 , a lin-

Table 1. Survey size and net usage.

\begin{tabular}{|c|c|c|c|c|c|c|c|}
\hline & \multicolumn{2}{|c|}{2009} & \multicolumn{2}{|c|}{2010} & \multicolumn{2}{|c|}{2011} & \multirow[t]{2}{*}{ Tota } \\
\hline & n & $\%$ & n & $\%$ & n & $\%$ & \\
\hline Individuals & 392 & - & 417 & - & 438 & - & 1247 \\
\hline Malaria (+) & 62 & 15.8 & 27 & 6.5 & 47 & 10.7 & - \\
\hline Net usage-total & 80 & 20.4 & 160 & 38.4 & 288 & 65.8 & - \\
\hline Net usage-malaria (+) & 6 & 9.7 & 6 & 22.2 & 26 & 55.3 & - \\
\hline Households & 81 & - & 98 & - & 106 & - & 285 \\
\hline Household net ownership & 41 & 50.6 & 69 & 70.4 & 101 & 95.3 & - \\
\hline
\end{tabular}


ear trend significant at the 0.10 level but not the 0.05 level, $r(1)=0.993, P=0.76$ (Figure 1). The largest increase in net usage was seen in participants over age 5 (Figure 2). The point prevalence of malaria decreased from $15.8 \%$ in 2009 to $6.5 \%$ in 2010 , and increased from $6.5 \%$ in 2010 to $10.7 \%$ in 2011 ; there was no linear trend between point prevalence of malaria and year, $r(1)=-0.547, \mathrm{P}=0.63$ (Figure 1). Children between ages 5 and 18 had the highest prevalence of malaria (Figure 3). Household net ownership increased linearly over the three years, from $50.6 \%$ in 2009 to $70.4 \%$ in 2010 , and then to $95.3 \%$ in 2011 (Table 1), $r(1)=0.998, \mathrm{P}=0.042$ (Figure 1).

The budget for the direct costs of the final year of the study was 2489 USD, with major expenses being transportation, personnel payment, and the purchase of the RDTs (Table 2). This amounts to a per-house cost of 23.50 USD.

\section{Discussion}

This paper describes a methodology for measuring bednet usage rates and malaria prevalence in a rural village in northwestern Tanzania at the household level over multiple consecutive years. The methodology examined in this paper allowed for more rapid and frequent surveys, as well as collection of additional data beyond bednet ownership and usage. Planning for the study was performed at the researchers' home institution in the months preceding the survey. The methodology required that researchers be on site for less than a month. One major advantage of this survey methodology is that it included actual malaria point prevalence in the village being surveyed, while the post-intervention monitoring program assessed only bednet distribution coverage and usage. When monitoring malaria vector control programs, actual malaria reduction must be assessed so that discrepancies in measures such as increased ITN usage and the expected malaria reduction can be immediately evaluated and addressed. An example is our finding that despite an increase in household net ownership and individual net usage over the three years, malaria prevalence did not significantly decrease. It is important to note that this study design is not a randomized trial with a control, so it would not be possible to identify bednet use or disuse as a direct cause of any change in malaria prevalence. However, this information gives health officials the opportunity to monitor the community's malaria status and to identify and address other factors that could account for changes in malaria prevalence. Though there are natural fluctuations in malaria prevalence from year to year, it is helpful to know whether any increase in malaria is in the setting of people not owning or using bednets, in which case further distribution and edu-

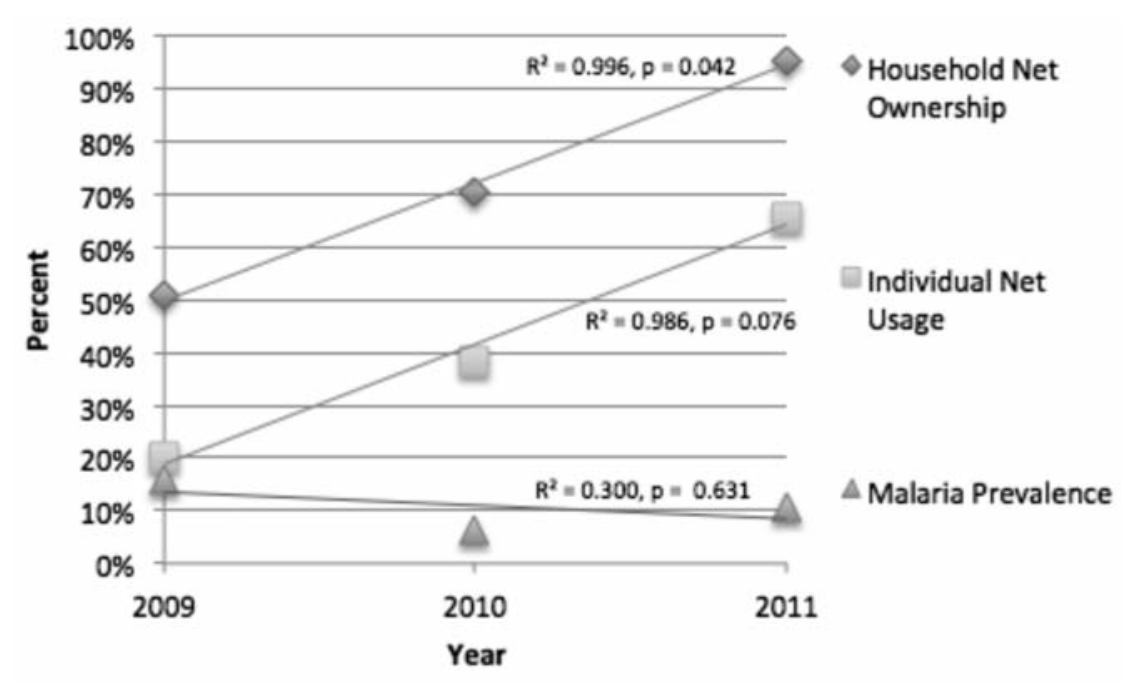

Figure 1. Malaria prevalence, net usage and household net ownership trends by year.

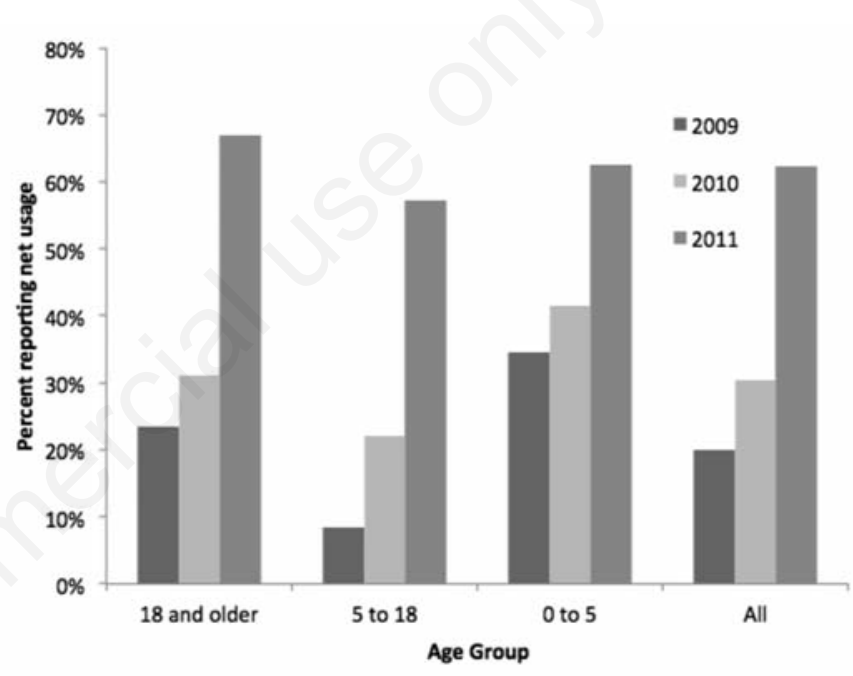

Figure 2. Bednet usage by age.

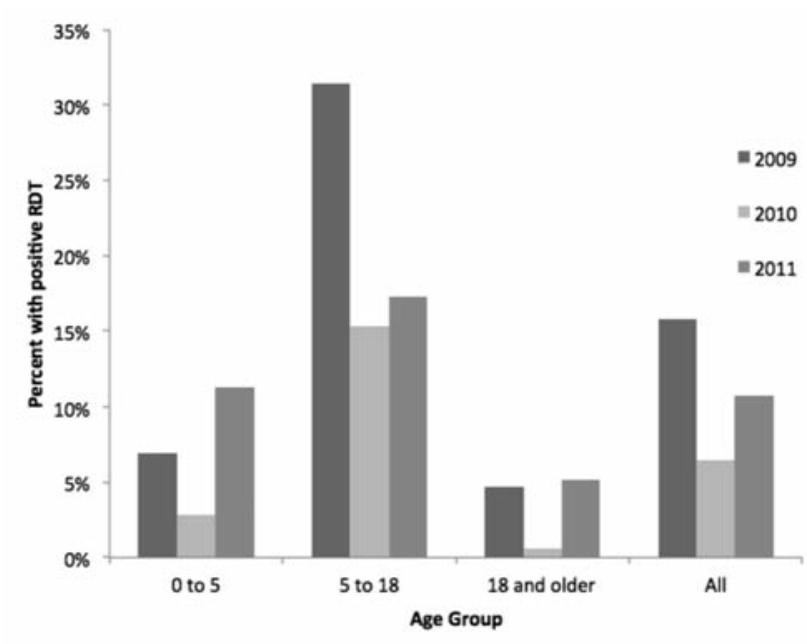

Figure 3. Malaria prevalence by age. 
Table 2. Study expenses.

\begin{tabular}{lcl} 
Items & Cost (USD) \\
Travel & & \\
In-country travel (bus, ferry) & 68 & Transport from Arusha to study site, 2 investigators \\
Total & 68 & - \\
Study materials & & \\
$\quad$ Miscellaneous expenses & 139 & Gloves, paracetamol, food and water for personnel \\
Rapid diagnostic test & 609 & 500 tests at 0.85 per test (plus shipping) \\
Artemisinin combination therapy & 139 & 100 doses \\
Total & 887 & - \\
\hline Personnel and vehicle & & \\
Nurses (2) & 209 & 10 days per nurse \\
Translators (2) & 558 & 10 days per translator \\
Guide (2) & 70 & Lump sum \\
Vehicle rental & 418 & 10 days in total \\
Total & 1255 & - \\
NIMR application fee & 279 & - \\
\hline Total & 2489 & - \\
\hline
\end{tabular}

NIMR, National Institute for Medical Research.

cation efforts can be undertaken, or if there are other unmet needs in terms of prevention strategies that should be addressed. The post-intervention monitoring program's methodology does not allow for such analysis, since malaria prevalence testing is not coupled with the bednet distribution evaluation.

Cost is often a major barrier to conducting a thorough evaluation of public health interventions. This survey cost roughly the same amount per household surveyed (23.50 USD per household) as the post-intervention monitoring program. However, our survey included the additional information of malaria prevalence at the household level. Furthermore, our survey was conducted in only one village while the postintervention monitoring program surveyed a total of 4500 households randomly selected from 150 villages in 5 representative districts. ${ }^{10}$ Our methodology would be expected to yield an even lower per-household cost if the evaluation was expanded to include more households because greater quantities of supplies such as the rapid antigen tests and the malaria treatment can be obtained at a lower unit price. This methodology can be replicated and scaled up into a cluster design to survey a larger proportion of the population. Expansion would be expected to lead to lower per-household costs through increasing economies of scale, as discussed previously. Additionally, the need for the researchers' presence at every household surveyed could be eliminated by training additional local personnel to conduct surveys independently, with great potential for partnership with students and faculty from Tanzanian universities and medical schools.

This study has several limitations. Due to its small size it may not be representative of what can be accomplished in other areas. Furthermore, as only one village that had received bednet distribution was surveyed, there was no chance for comparison with other villages without bednet distribution which could have added information about natural variation in malaria. Our sampling method discluded people who were not home at the time of the survey, possibly skewing our sample as people who spend their days outside their households may have different risk factors and therefore prevalence of malaria. The data collected about bednet usage may not be accurate if participants were not truthful about their bednet usage, although we tried to eliminate this effect by observing the bednets hanging up. The survey did not account for behaviors such as time out of net at night, proper use, or net quality, which can influence the effectiveness of bednets in preventing malaria.

\section{Conclusions}

Evaluations of bednet distribution programs must measure their degree of success in reaching their fundamental goal, endpoint reduction achieved quickly and cost-effectively through randomized household surveys measuring bednet usage and malaria prevalence at the household level. Although our study was comparatively small, covering just one village, we believe that it demonstrates the feasibility of conducting an evaluation of a bednet distribution campaign that links bednet ownership and usage data to malaria prevalence, which is ultimately the intended target of such an intervention.

\section{References}

1. WHO. World malaria report. Geneva, in malaria prevalence. We argue that this can be
Switzerland: World Health Organization; 2010.

2. PMI. Malaria operational plan. Dar es Salaam, Tanzania: President's Malaria Initiative; 2012. Available from: http:/www.pmi.gov/countries/profiles/tanzania.html

3. Hanson K, Nathan R, Marchant T, et al. Vouchers for scaling up insecticide-treated nets in Tanzania: methods for monitoring and evaluation of a national health system intervention. BMC Public Health 2008;8:205.

4. Sexton AR. Best practices for insecticidetreated bed netbednet distribution programme in sub-Saharan eastern Africa. Malaria J 2011;10:157.

5. WHO. World malaria report. Geneva, Switzerland: World Health Organization; 2009.

6. Vanden Eng JL, Thwing J, Wolkon A, et al. Assessing bed net use and non-use after long-lastig insecticidal net distribution: a simple framework to guide programmatic strategies. Malaria J 2010;9:133.

7. Hanson K, Marchant T, Nathan R, et al. Household ownership and use of insecticide treated nets among target groups after implementation of a national voucher programme in the United Republic of Tanzania: plausibility study using three annual cross sectional household surveys. Brit Med J 2009;339:2434.

8. Beier JC, Keating J, Githure JI, et al. Integrated vector management for malaria control. Malaria J 2008;7:S4.

9. Lindblade KA, Eisele TP, Gimnig JE, et al. Sustainability of reductions in malaria transmission and infant mortality in western Kenya with use of insecticide-treated bednets: 4 to 6 years of follow-up. JAMA-J Am Med Assoc 2004;291:2571-80.

10. Bonner K, Mwita A, McElroy PD, et al. Design, implementation and evaluation of a national campaign to distribute nine million free LLINs to children under five years of age in Tanzania. Malaria J 2011;10:73.

11. TACAIDS. Tanzania HIV/AIDS and malaria indicator survey 2007-08. Dar es Salaam, Tanzania: Tanzania Comission for AIDS; 2008.

12. WHO. Malaria rapid diagnostic test performance: summary results of WHO product testing of malaria RDTs: rounds 1 and 2. Geneva, Switzerland: World Health Organization on behalf of the Special Programme for Research and Training in Tropical Diseases; 2009.

13. WHO. Malaria indicator survey: basic documentation for survey design and implementation. Geneva, Switzerland: World Health Organization Roll Back Malaria Monitoring and Evaluation Reference Group; 2005. 